\title{
The study of drug resistance properties of ABCG2 (ATP- binding cassette G2) in contact with thymoquinone, gallic acid, and hesperetin antioxidants
}

\author{
Javad Saffari-Chaleshtori ${ }^{1,2}$, Sayed Mohammad Shafiee ${ }^{1},{\text { Keihan Ghatreh-Samani }{ }^{3 *} \text {, Narges Jalilian }}^{4}$ \\ ${ }^{1}$ Department of Biochemistry, School of Medicine, Shiraz University of Medical Sciences, Shiraz, Iran \\ ${ }^{2}$ Student Research Committee, Shiraz University of Medical Sciences, Shiraz, Iran \\ ${ }^{3}$ Clinical Biochemistry Research Center, Basic Health Sciences Institute, Shahrekord University of Medical Sciences, Shahrekord, Iran \\ ${ }^{4}$ Medical Plants Research Center, Basic Health Sciences Institute, Shahrekord University of Medical Sciences, Shahrekord, Iran
}

\section{A R T I C L E I N F O}

Article Type:

Original Article

Article History:

Received: 12 May 2018

Accepted: 24 December 2018

\section{Keywords:}

Antioxidant

ABCG2 transporter

Cancer cell

Simulation

\begin{abstract}
A B S T R A C T
Introduction: ATP-binding cassette $(\mathrm{ABC})$ transporters are a group of intra membrane proteins that play key roles in the transmission and exchange of vital compounds on both sides of the membrane. These proteins can specially transport anti-cancer drugs out of cancer cells. ABCG2 is a member of this family that is extremely expressed in many cancers. This study, aims to evaluate the binding affinity of three antioxidants thymoquinone (TQ), gallic acid (GA), and hesperetin (HP) to ABCG2 compared with an anti-cancer drug, mitoxantrone (Mit), to export cells.

Methods: The PDB file of ABCG2 was obtained from the protein data bank server (http:// www.rcsb.org) with ID: 5NJ3. After 200 stages of molecular docking running on ABCG2 protein in AutoDock v.4.2 software, the amino acids involved in the binding site of each compound were identified using the $\mathrm{LigPlot}^{+}$software.

Results: HP had the lowest $(-6.36 \mathrm{kcal} / \mathrm{mol})$ and GA had the highest $(-3.93 \mathrm{kcal} / \mathrm{mol})$ binding energy in comparison with Mit $(-0.06 \mathrm{kcal} / \mathrm{mol})$ for binding to ABCG2. Effective concentration required to perform the reaction between ABCG2 was higher in GA (1.31 mM) than TQ $(42.69 \mu \mathrm{M})$ and HP $(21.74 \mu \mathrm{M})$. GA, HP, and TQ formed 17, 18, and 22 hydrogen and hydrophobic bonds at the binding site of ABCG2.

Conclusion: It seems that GA has the lowest affinity to make contact with ABCG2 binding site. So, GA tends to remain in the cell but TQ and HP tend to leave the cell easily via ABCG2 transporter.
\end{abstract}

Implication for health policy/practice/research/medical education:

Gallic acid is an antioxidant that has the low tendency for expose out of the cells. It as an effective antioxidant is better than TQ and HP for treatment of cancers.

Please cite this paper as: Saffari-Chaleshtori J, Shafiee SM, Ghatreh-Samani K, Jalilian N. The study of drug resistance properties of ABCG2 (ATP-binding cassette G2) in contact with thymoquinone, gallic acid, and hesperetin antioxidants. J Herbmed Pharmacol. 2019;82):108-113. doi: 10.15171/jhp.2019.17.

\section{Introduction}

ATP-binding cassette $(\mathrm{ABC})$ transporters are a family of proteins that are present in cell membrane and play key roles in the transport of vital compounds out of the cells (1). There are $48 \mathrm{ABC}$ transporter proteins, known in human body cells, which are divided into seven subgroups of $A B C$ (A-G) based on their size, number of subunits and their building (2,3). Any malfunctioning of these membrane proteins causes a variety of diseases and the occurrence of mutations in the building of these molecules also causes genetic disorders such as cystic fibrosis (4), gout (5) and lipid metabolic abnormalities (6). Today, the importance of these membrane transporters has increased due to their role in resistance to anticancer drugs that cause the drug to escape from the cell before it exerts its effect (7). ABCG2 transporters are of the most important members of this great family. They exist and have physiological roles in many tissues, such as breast, brain, and testicular, as well as in many cancers, especially breast cancer, stomach cancer, and pancreatic cancer (8-10). The most important 
physiological role of $\mathrm{ABCG}_{2}$ transporter is the transfer of uric acid. Any disruption in the normal functioning of this protein causes diseases such as gout, hypertension, and kidney disorders (11). In addition, its expression is increased in many cancers and can be recognized as a marker of cancer at the cell surface. However, it is a proprietary marker for hematopoietic stem cells (12). $\mathrm{ABCG}_{2}$ as an intracellular drug transporter, causes the drug resistance in cancer cells (13). Despite significant advances in the treatment of various types of cancers, there is still an increasing incidence of cancer in different societies $(14,15)$. On the other hand, studies have shown that herbal compounds play a very important role in the death of different types of cancer cells (16). Hesperetin (HP) or 2,3-Dihydro-5,7-dihydroxy-2- (3-hydroxy-4methoxyphenyl)-4H-1 benzopyran-4-one), gallic acid (GA) or 3,4,5-trihydroxybenzoic acid and thymoquinone (TQ) or 2-Isopropyl-5-methylbenzo-1,4-quinone $(17,18)$ are effective compounds found in plants such as onion, apple, green and black tea, red grapes, citrus, broccoli sprouts, tomato (19) and in some fruits like berries and grapes, hard wood plant species such as chestnut (Castanea sativa L.) and oak (Quercus robur), and also in tea (20) and Nigella sativa Linn (21). Many studies have shown that these three active plant compounds are very effective in destroying cancer cells, especially gastric cancer cells (22-24). Because of the importance of ABC transfer proteins in cancer cells, especially gastric cancer cells, as well as the confirmation of the effects of HP, GA, and TQ antioxidants on cell death in gastric cancer cells. This study, was designed to evaluate the effects of these three antioxidants on ABCG2 transporter using simulation and molecular docking and measuring the tendency of this protein to remove these three plant compounds from the cell, compared to anticancer drug, mitoxantrone (Mit).

\section{Materials and Methods}

We obtained the ABCG2 PDB file (ID: 5NJ3) from protein data bank server (http://www.rcsb.org) and optimized with Arguslab v.4.0 software. Then, we obtained the antioxidant files of GA (CID:370), HP (CID: 72281), and TQ (CID:10281) from Pubchem server and converted them to PDB files and optimized using Arguslab v.4.0 software (25).

Molecular docking studies were done by Autodock v.4.2 software in Linux operating system by a 64-bit system with Intel ${ }^{\circledR}$ Core $^{\mathrm{TM}}$ i7 CPU Server in Clinical Biochemistry Research Center, Basic Health Sciences Institute of Shahrekord University of Medical Sciences.

Molecular docking of GA, HP, and TQ on ABCG2 transporter was done to find the best binding sites for the ligand-receptor and to determine the most stable free energy state of ligand-receptor. In this study, we built a Grid Box with $60 \times 120 \times 72 \mathrm{~nm}(\mathrm{x} \times \mathrm{y} \times \mathrm{z})$ for intra cellular NBD domains, after the production of PDBQ and PDBQT files of TQ, GA, and HP as ligands and ABCG2 transporter as receptor. We used autogrid $4-p$ n.gpf $-l n$.gle linux in order to produce the n.gle text file. After 200 stages of molecular docking running on ligands, we used genetic algorithm (GA) and Lamarckian GA parameters. To produce the n.dlg text file, we used autodock $4-p$ n.dpf $-l$ n.dlg linux order. The data obtained from n.dlg file, were analyzed (26).

In this study, we used LigPlot ${ }^{+}$software to specify the number of hydrophobic and hydrogen bonds between ABCG2 transporter and GA, TQ, and HP ligands and the type and number of amino acids present in the binding site were identified (27).

\section{Results}

Figure 1 shows the structure of ABCG2 transporter and three antioxidants HP, GA, and TQ. In this figure, nucleotide binding domains (NBDs) in intra cellular membrane and transmembrane domains (TMDs) in extra cellular membrane were visible.

Table 1 shows the molecular interaction and the binding energies of Mit, GA, HP, TQ, with ABCG2. After Mit, the estimated binding free energy (BE) for GA was higher than HP and TQ. Estimated inhibition constant (EIC) for GA was lower than HP and TQ.

Figure 2 shows that the interaction between ABCG2 with TQ has the most hydrogen and hydrophobic bonds and after the interaction between ABCG2 with Mit, interaction between ABCG2 with GA has the least hydrogen and hydrophobic bonds.

\section{Discussion}

The potential of stem cells in the tissues and the ability to differentiate into somatic cell types have played very important roles in the reconstruction of damaged tissue parts $(28,29)$. Cancer stem cells (CSCs), which in many

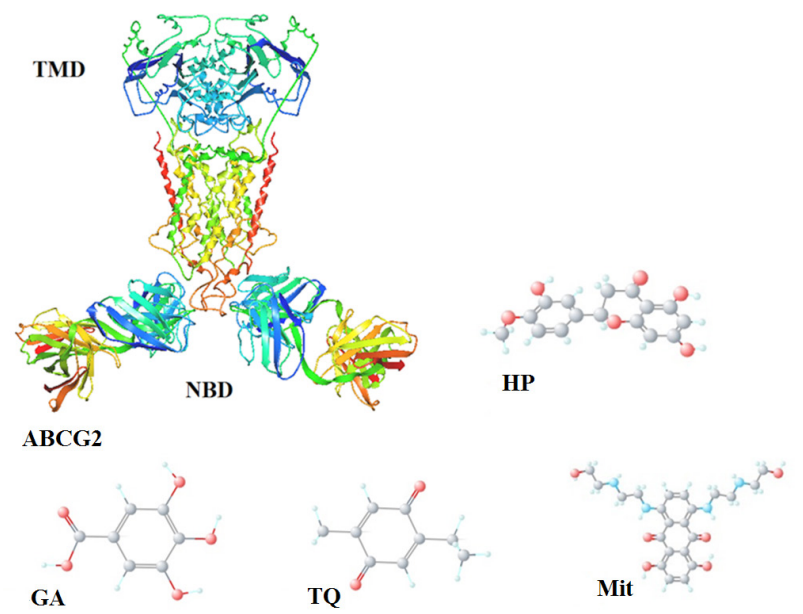

Figure 1. The structure of molecules. ABCG2 transporter with nucleotide binding domains (NBDs) and transmembrane domains (TMDs). HP: hesperetin, GA: gallic acid, TQ: thymoquinone, Mit: mitoxantrone. 
Table 1. Molecular docking parameters between ABCG2 transporter and other ligands

\begin{tabular}{|c|c|c|c|c|c|}
\hline \multicolumn{2}{|c|}{ Interaction bonds } & \multirow{2}{*}{ EIC } & \multirow{2}{*}{ FIE } & \multirow{2}{*}{ BE } & \multirow{2}{*}{ Ligands } \\
\hline Hydrophobic Bonding & Hydrogen Bonding & & & & \\
\hline $\begin{array}{l}\text { Val1802, Ser1803, Leu1804, Gly1805, } \\
\text { Asp1806 }\end{array}$ & Val1802, Ser1803, Leu1804, Gly1805, Asp1806 & $900.1 \mathrm{mM}$ & -4.48 & $0.06-$ & Mit \\
\hline $\begin{array}{l}\text { Asp419, Tyr1462, Arg1403, Phe1262, } \\
\text { Gly1264, Tyr 1166, Thr1465 }\end{array}$ & $\begin{array}{l}\text { Ser420, Trp1463, Asn1165, Asp419, Tyr1462, } \\
\text { Arg1403, Phe1262, Gly1264, Tyr 1166, Thr1465 }\end{array}$ & $1.31 \mathrm{mM}$ & -5.72 & -3.93 & GA \\
\hline $\begin{array}{l}\text { Gln1584, Gln1692, Asn1831, Lys1828, } \\
\text { Arg1834, Ala1832, Tyr1689, Leu1585, } \\
\text { Trp1690 }\end{array}$ & $\begin{array}{l}\text { Gln1584, Gln1692, Asn1831, Lys1828, Arg1834, } \\
\text { Ala1832, Tyr1689, Leu1585, Trp1690 }\end{array}$ & $21.74 \mu \mathrm{M}$ & -6.96 & -6.36 & $\mathrm{Hp}$ \\
\hline $\begin{array}{l}\text { Tyr51, Ser420, Asn1165, Asn419, Trp1467, } \\
\text { Thr1465, Pro593, Tyr1462, Trp1463, } \\
\text { Arg1403, Tyr1166 }\end{array}$ & $\begin{array}{l}\text { Tyr51, Ser420, Asn1165, Asn419, Trp1467, } \\
\text { Thr1465, Pro593, Tyr1462, Trp1463, Arg1403, } \\
\text { Tyr1166 }\end{array}$ & $42.69 \mu \mathrm{M}$ & -6.26 & -5.96 & TQ \\
\hline
\end{tabular}

Abbreviations: BE, Estimated Free Energy of binding (kcal/mol); FIE, final intermolecular energy (kcal/mol); EIC, estimated inhibition constant; Mit, mitoxantrone; $\mathrm{GA}$, gallic acid; $\mathrm{HP}$, hesperetin; $\mathrm{TQ}$, thymoquinone.

cases originate from stem cells, also behave like stem cells. They have the ability to grow, replicate, and differentiate into other cancer cell lines (30-33). However, studies have shown that, at the CSCs surface, cell-specific markers increase expression, which can be specific for each cell line. For example, in GCSC cells, ABCB1 cell markers, CD133, ABCG2, are strongly expressed (9). Increase in expression of $\mathrm{ABC}$ carriers induces a unique feature in GCSC cells, which makes them resistant to chemotherapy (34). Nowadays, the structure and function of $A B C$ transporter proteins are well known. This family of proteins consists of two domains, NBDs with ATPase activity inside the cell and two TMDs attached to the outer membrane $(35,36)$. Our study well illustrated that the ABCG2 transporter had a great ability to convey different compounds out of the cell. The strong tendency of GA, TQ, and QC toward the binding site in the NBD domains represents a tendency for this transition. Recent studies have shown that the amino acid E446 in NBD domain of ABCG2 transfer, plays an important role in the transfer of drugs to the outside of
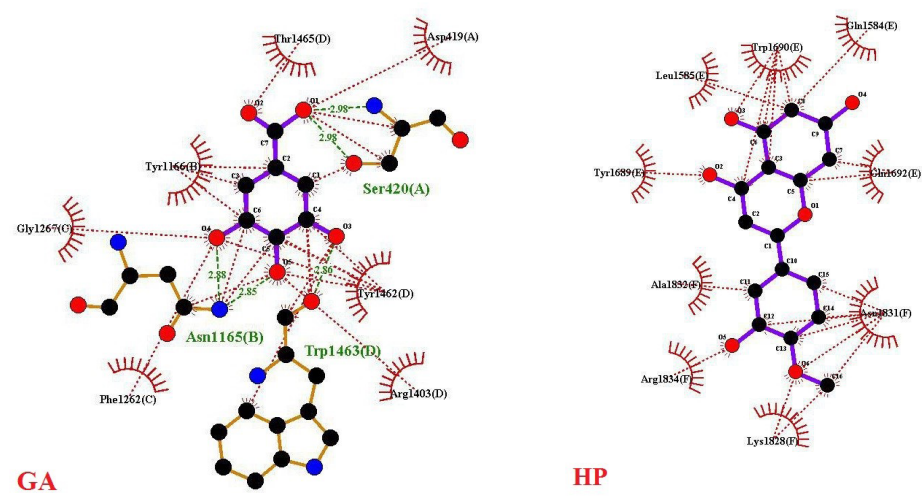

HP
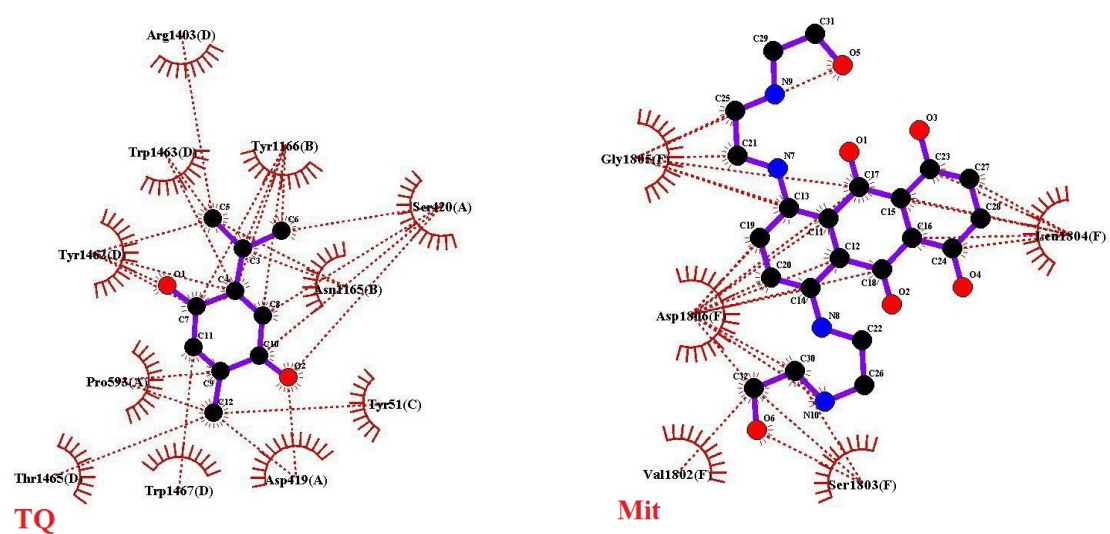

Figure 2. Interaction bonds such as hydrogen and hydrophobic bonds between ABCG2 with Mit, GA, HP and TQ. Mit, mitoxantrone; GA, gallic acid; HP, hesperetin; TQ, thymoquinone. 
the cell as well as the ATPase property of this transporter. So that the removal of any of the E466K, E451R, E458R D477R amino acids causes a disruption of protein function and the deficiency of efflux of the drug Mit from cell. Also, K647, K652, K653 amino acids in the TMD domain interact with cellular membrane phospholipids electro statically, which stabilizes the protein and binds to the cell membrane. However, they don't have a significant role in the efflux of Mit drug (37). Other studies have shown that the amino acid K473 plays an important role in the formation and deployment of monomers alongside each other (38-40). The ABCG2 transporter plays an important role in the efflux of cholesterol from the cell. Taylor et al. showed that six amino acids F432, F439, L539, I543, V546, and M549 play a key role in connecting two cholesterol molecules to the ABCG2 molecule (41). The fluorescent dyes Hoechst 33342 and rhodamine123 (R123) are desire substrates for the ABCG2 and ABCB1 transporters, respectively (42). In our study, Figure 2 and Table 1 show that anti-drug cancer Mit, just interacted with five amino acids (Val1802, Ser1803, Leu1804, Gly1805, Asp1806) in binding site. However, the highest amount of estimated binding free-energy $(\mathrm{BE})$ was $-0.06 \mathrm{~kJ} / \mathrm{mol}$ and the highest EIC was $900.1 \mathrm{mM}$. This indicates that the rate at which Mit binds to the binding site in the ABCG2 molecule is very low and the possibility of efflux of this drug from the cell by this transporter is very low. In addition, in order to allow this connection to be thermodynamically applicable, the Mit concentration of the cell should reach $900.1 \mathrm{mM}$. This concentration of drug is very high. However, at much lower concentrations, the drug can play its role in the treatment process in cancer cells. Many studies have shown that GA is a powerful antioxidant compound in the death of cancer cells $(43,44)$.

Based on the results in Figure 2 and Table 1, GA interacts with amino acids in binding site by ten hydrogen bonds and six hydrophobic bonds. Its BE was $-3.93 \mathrm{kcal} / \mathrm{mol}$ and its EIC was $1.31 \mathrm{mM}$. These amounts are lower than the amounts obtained from Mit connection with the binding site. However, compared to the two anti-oxidants, HP and TQ are much higher, such that HP and TQ had 18 and 22 interaction bonds with ABCG2 and their BE were -6.96 and $-6.36 \mathrm{kcal} / \mathrm{mol}$. HP and TQ with lower concentrations of 21.74 and $42.69 \mu \mathrm{M}$ were in contact with ABCG2. This indicates that they tend to efflux the cell. Previous studies have shown that the $\mathrm{IC}_{50}$ concentrations of these three antioxidant compounds are different for the induction of anticancer effects in different cancer cells. The lowest $\mathrm{IC}_{50}$ has been obtained in comparison with HP and TQ for the GA molecule $(25,45,46)$. This can be a reason for the results obtained in this study. Our study showed that GA compared to other two compounds could be more resistant to low concentrations in cancer cells, hence, it exerts its anticancer effects, while two other drugs (TQ and HP) need to use much higher concentrations, which can have toxic effect on other cells.

\section{Conclusion}

Although antioxidant compounds can inhibit cancer cells and induce cancer cell death, but proprietary transponders, such as ABCG2, discard these compounds and other anticancer drug compounds from cancer cells. This study showed that GA, compared with TQ and HP by a very low binding affinity with these membrane transfer proteins, could have longer existence in the cell, and would have a higher effect on the removal of cancer cells.

\section{Authors' contributions}

SMS and KGS designed and managed the study. JSC and NJ carried out the molecular dynamic studies. JSC prepared the draft. All authors read and approved the final version of the manuscript.

\section{Conflict of interests}

Authors declare that there is not any conflict of interest.

\section{Ethical considerations}

Ethical issues including text plagiarism, misconduct, manipulation or appropriation, data fabrication, falsification, redundant publication as well as duplicate submissions have been carefully observed by authors.

\section{Funding/Support}

This study was funded by the Deputy of Research and Technology of the Shahrekord University of Medical Sciences, Shahrekord, Iran, based on the research project number: 267.

\section{References}

1. Kuchler $\mathrm{K}$. The $\mathrm{ABC}$ of $\mathrm{ABCs}$ : multidrug resistance and genetic diseases. FEBS J. 2011;278(18):31-89. doi: 10.1111/j.1742-4658.2011.08234.x.

2. Moitra K, Dean M. Evolution of ABC transporters by gene duplication and their role in human disease. Biol Chem. 2011;392(1-2):29-37.

3. Dean M, Hamon Y, Chimini G. The human ATP-binding cassette (ABC) transporter superfamily. J Lipid Res. 2001;42(7):1007-17.

4. Riordan JR, Rommens JM, Kerem BS, Alon N, Rozmahel R, Grzelczak Z, et al. Identification of the cystic fibrosis gene: cloning and characterization of complementary DNA. Science. 1989;245(4922):1066-73.

5. Woodward OM, Köttgen A, Coresh J, BoerwinkleE, Guggino WB, Köttgen M. Identification of a urate transporter, ABCG2, with a common functional polymorphism causing gout. Proc Nat Acad Sci. 2009;106(25):10338-42. doi: 10.1073/pnas.0901249106.

6. Westerterp M, Bochem AE, Yvan-Charvet L, Murphy AJ, Wang N, Tall AR. ATP-binding cassette transporters, atherosclerosis, and inflammation. Circul Res. 2014;1170157:(1) 4

7. Glavinas H, Krajcsi P, Cserepes J, Sarkadi B. The role of 
$\mathrm{ABC}$ transporters in drug resistance, metabolism and toxicity. Curr Drug Deliv. 2004;1(1):27-42.

8. Cuperus FJ, Claudel T, Gautherot J, Halilbasic E, Trauner $\mathrm{M}$. The role of canalicular ABC transporters in cholestasis. Drug Metab Dispos. 2014;42(4):546-60. doi: 10.1124/ dmd.113.056358.

9. Jiang Y, He Y, Li H, Li H-N, Zhang L, Hu W, et al. Expressions of putative cancer stem cell markers ABCB1, ABCG2, and CD133 are correlated with the degree ofdifferentiation of gastric cancer. Gastric Cancer. 2012;15(4):440-50.

10. Diestra JE, Scheffer GL, Catala I, Maliepaard M, Schellens $\mathrm{JH}$, Scheper RJ, et al. Frequent expression of the multi-drug resistance-associated protein BCRP/MXR/ABCP/ABCG2 in human tumours detected by the BXP-21 monoclonal antibody in paraffin-embedded material. J Pathol. 2002;198(2):213-9.

11. Ishikawa T, Aw W, Kaneko K. Metabolic interactions of purine derivatives with human $\mathrm{ABC}$ transporter ABCG2: genetic testing toassess gout risk. Pharmaceuticals. 2013;6(11):1347-60. doi: 10.3390/ph6111347.

12. Zhou S, Schuetz JD, Bunting KD, Colapietro A-M, Sampath J, Morris JJ, et al. The ABC transporter Bcrp1/ABCG2 is expressed in a wide variety of stem cells and is a molecular determinant of the side-population phenotype. Nat Med. 2001;7(9):1028.

13. Sharom FJ. ABC multidrug transporters: structure, function and role in chemoresistance. Pharmacogenomics. 2008;9(1):105-27.

14. Malekzadeh R, Derakhshan MH, Malekzadeh Z. Gastric cancer in Iran: epidemiology and risk factors .Arch Iran Med. 2009;12(6):576-83.

15. Saffari-Chaleshtori J, Tabatabaiefar M-A, GhasemiDehkordi P, Farokhi E, Moradi M-T, HashemzadehChaleshtori M. The lack of correlation between TP53 mutations and gastric cancer: a report from a province of Iran. Genetika Belgrade. 2017;49(1):2.46-3535

16. Eskandari E, Heidarian E, Amini SA, Saffari-Chaleshtori J. Evaluating the effects of ellagic acid on pSTAT3, pAKT, and pERK1/2 signaling pathways in prostate cancer PC3 cells. J Cancer Res Ther. 2016;12(4):1266. doi: 10.4103/09731482.165873.

17. Locatelli C, Filippin-Monteiro FB, Creczynski-Pasa TB. Alkyl esters of gallic acid as anticancer agents: a review. Eur J Med Chem. 2013;60:233-9.

18. Suguna P, Geetha A, Aruna R, Siva GV. Effect of thymoquinone on ethanol and high fat diet induced chronic pancreatitis-a dose response study in rats. Indian J Exp Biol. 2013;51(4):292-302.

19. Arts MJ, Dallinga JS, Voss HP, Haenen GR, Bast A. A new approach to assess the total antioxidant capacity using the TEAC assay. Food Chem. 2004;88(4):567-70.

20. Singh J ,Rai G, Upadhyay A, Kumar R, Singh K. Antloxldant phytochemicals in tomato (Lycopersicon esculentum). Indian J Agr Sci. 2004;74(1):3-5.

21. Ali B, Blunden G. Pharmacological and toxicological properties of Nigella sativa. Phytother Res. 2003;17(4):299305.

22. Haghi A, Azimi H, Rahimi R. A comprehensive review on pharmacotherapeutics of three phytochemicals, curcumin, quercetin, and allicin, in the treatment of gastric cancer. J Gastrointest Cancer. 2017;48(4):314-320. doi: 10.1007/ s12029-017-9997-7.

23. Ryu B, Kim S-Y, Vo TS, Kim WS, Kim DG, Kim SK. Characterization of the in vitro effects of gallic acidgrafted-chitooligosaccharides in the suppression of AGS human gastric cancer cell proliferation. RSC Adv. 2017;7(39):24561-8. doi: 10.1039/C7RA02487H.

24. FengL-M, Wang X-F, Huang Q-X. Thymoquinone induces cytotoxicity and reprogramming of EMT in gastric cancer cells by targeting PI3K/Akt/mTOR pathway. J Biosci. 2017;42(4):547-54.

25. Saffari-Chaleshtori J, Heidari-Sureshjani E, Moradi F, Jazi HM, Heidarian E. The study of apoptosis-inducing effects of three pre-apoptotic factors by gallic acid, using simulation analysis and the comet assay technique on the prostatic cancer cell line PC3. Malaysian J Med Sci. 2017;24(4).18

26. Chaleshtori JS, Soreshjani EH, Reisi F, Tabatabaiefar MA, Asadi-Samani M, Navid Z, et al. Damage intensity of carvacrol on prostatic cancer cells lineDu145 and molecular dynamic simulation of it effect on apoptotic factors. Int J Pharm Tech Res. 2016;9(6):261-73.

27. Saffari-Chaleshtori J, Heidari-Soreshjani E, AsadiSamani M. Computational study of quercetin effect on pre-apoptotic factors of Bad, Bak and Bim. J Herbmed Pharmacol. 2016;5(2):61-66.

28. Daley GQ. The promise and perils of stem cell therapeutics. Cell Stem Cell. 2012;10(6):740-9.

29. Ram Singh S. Stem cell niche in tissue homeostasis, aging and cancer. Curr Med Chem. 2012;19(35):5965-74.

30. Borovski T, Felipe De Sousa EM, Vermeulen L, MedemaJP. Cancer stem cell niche: the place to be. Cancer Res. 2011;71(3):634-9. doi: 10.1158/0008-5472.CAN-10-3220.

31. Bomken S, Fišer $\mathrm{K}$, Heidenreich $\mathrm{O}$, Vormoor J. Understanding the cancer stem cell. Bri J Cancer. $2010 ; 103(4): 439$.

32. Varon C, Dubus P, Mazurier F, Asencio C, Chambonnier L, Ferrand J, et al. Helicobacter pylori infection recruits bone marrow- derived cells that participate in gastric preneoplasia in mice. Gastroenterology. 2012;142(2):28191. doi: 10.1053/j.gastro.2011.10.036

33. Barker N, Bartfeld S, Clevers H. Tissue-resident adult stem cellpopulations of rapidly self-renewing organs. Cell Stem Cell. 2010;7(6):656-70.

34. Dean M. ABC transporters, drug resistance, and cancer stem cells. J Mammary Gland Biol Neoplasia. 2009;14(1):39.

35. Linton KJ, Higgins CF. Structure andfunction of ABC transporters: the ATP switch provides flexible control. Pflügers Arch. 2007;453(5):555-67.

36. Kerr ID. Structure and association of ATP-binding cassette transporter nucleotide-binding domains. Biochim Biophys Acta Biomembr. 2002;1561(1):47-64.

37. Khunweeraphong N, Stockner T, Kuchler K. The structure of the human $\mathrm{ABC}$ transporter ABCG2 reveals a novel mechanism for drug extrusion. Sci Rep. 2017;7(1):13767. doi: 10.1038/s41598-017-11794-w.

38. Haider AJ, Cox MH, Jones N, Goode AJ, Bridge KS, Wong $\mathrm{K}$, et al. Identification of residues in ABCG2 
affecting protein trafficking and drug transport, using co-evolutionary analysis of ABCG sequences. Biosci Rep. 2015;35(4):e00241.

39. Woodward OM, Tukaye DN, CuiJ, Greenwell P, Constantoulakis LM, Parker BS, et al. Gout-causing Q141K mutation in ABCG2 leads to instability of the nucleotidebinding domain and can be corrected with small molecules. Proc Natl Acad Sci. 2013;110(13):5223. doi: 10.1073/ pnas. 1214530110.

40. Cai X, Bikadi Z, Ni Z, Lee E-W, Wang H, Rosenberg MF, et al. Role of basic residues within or near the predicted transmembrane helix 2 of the human breast cancer resistance protein in drug transport. J Pharmacol Exp Ther. 2010;333(3):670-81.

41. Taylor NM, Manolaridis I, Jackson SM, Kowal J, Stahlberg $\mathrm{H}$, Locher KP. Structure of the human multidrug transporter ABCG2. Nature. 2017;546(7659):504. doi: $10.1038 /$ nature22345.

42. Mulder A, Visser J. Separation and functional analysis of bone marrowcells separated by rhodamine-123 fluorescence. Exp Hematol. 1987;15(1):99-104.
43. Choi SY, Kee HJ, Jin L, Ryu Y, Sun S, Kim GR, et al. Inhibition of class IIa histone deacetylase activity by gallic acid, sulforaphane, TMP269, and panobinostat. Biomed Pharmacother. 2018;101:145-54. doi: 10.1016/j. biopha.2018.02.071.

44. Weng Y-P, Hung P-F, Ku W-Y, Chang C-Y, Wu B-H, Wu $\mathrm{M}-\mathrm{H}$, et al. The inhibitory activity of gallic acid against DNA methylation: application of gallic acid on epigenetic therapy of human cancers. Oncotarget. 2018;9(1):361. doi: 10.18632/oncotarget.23015.

45. Shirzad M, Heidarian E, Beshkar P, Gholami-Arjenaki M. Biological effects of hesperetin on interleukin-6/ phosphorylated signal transducer and activator of transcription 3 pathway signaling in prostate cancer PC3 cells. Pharmacogn Res. 2017;9(2):188. doi: 10.4103/09748490.204655.

46. Ranjbari A, Heidarian E, Ghatreh-Samani K. Effects of thymoquinone on IL-6 Gene expression and some cellular signaling pathways in prostate cancer PC3 cells. Jundishapur J Nat Pharm Prod. 2017;12(3):e63753. 